\title{
Gerbard Thür
}

\section{Armut \\ Gedanken zu Ehegüterrecht und Familienvermögen in der griechischen Polis}

Eine Bauernhochzeit ist auch heutzutage noch ein großes Ereignis. Vergnügt taucht der Städter in den Trubel ein; festliches Essen und Trinken, Blasmusik, Tanz, vielleicht sogar eine zünftige Rauferei. Gehört man dazu oder spielt man Folklore? Kaum ein Zaungast sieht, welch organisatorische Leistung nötig ist, um solch ein Fest mit ein- bis zweihundert Gästen auszurichten. Der moderne Mensch fragt sogleich nach den Kosten: Zahlt der Brautvater, zahlen beide Beteiligten gemeinsam oder wird auf

- Das der Spātantike und Byzanz gewidmete Colloquium bot Gelegenheit, die altgriechische Polis aus etwas größerem Abstand zu betrachten. Dies förderte die Neigung zur Generalisierung. Der Leser sei aber davor gewarnt, aus dem Beitrag etwa "Grundgedanken“ des altgriechischen Ehegüterrechts ableiten zu wollen. Dem skizzenhaften Charakter der Überlegungen entsprechend, behielt ich die Vortragsfassung bei. Genauere Information bieten die folgenden, zumeist neueren Abhandlungen, die auch die ältere Literatur erschließen (sie werden später nur noch mit dem Namen des Autors und, wenn nötig, mit der Jahreszahl zitiert): Karl-Dieter Albrecht, Rechtsprobleme in den Freilassungen der Böotier, Phoker, Dorier, Ost- und Westlokrer (Paderborn 1978); Antoine M. Babacos, Actes d'aliénation en commun et autres phénomènes apparentés d'après le droit de la Thessalie antique (Thessaloniki 1966); Antonios M. Babakos, Familienrechtliche Verhāltnisse auf der Insel Kalymnos im ersten nachchristlichen Jahrhundert (KölnWien 1973); Jacqueline Cbristien, La loi d'Epitadeus, in: RHD 52 (1974) 197-221; Carl Cromme, Personen- und Familiengüterrecht in den delphischen Freilassungsurkunden, in: RIDA 9 (1962) 177-238; Roland Étienne, Les femmes, la terre et l'argent à Ténos à l'époque hellénistique, in: La femme dans le monde méditerranéen, Bd. 1, Hrsg. A.-M. Vérilbac (Lyon 1985) 61-70; Lin Foxball, Household, Gender and Property in Classical Athens, in: ClQu 39 (1989) 22-44; Peter Garnsey, Famine and Food Supply in the Graeco-Roman World (Cambridge 1988); Günther Häge, Ehegüterrechtliche Verhāltnisse in den griechischen Papyri Ägyptens bis Diokletian (Köln-Graz 1968); A. R. W. Harrison, The Law of Athens, Bd. 1: The Family and Property (Oxford 1968); Stephen Hodkinson, Land Tenure and Inheritance in Classical Sparta, in: ClQu 36 (1986) 378-406; ders., Inheritance, Marriage and Demography: Perspectives upon the Success and Decline of Classical Sparta, in: Classical Sparta, Hrsg. A. Powell (London 1989) 79-121; Evanghelos Karabelias, L'épiclérat attique (thèse dactyl., Paris II, 1974); ders., Recherches sur la condition juridique et sociale de la fille unique dans le monde grec ancien excepté Athènes (dactyl., Paris 1980); ders., L'épiclérat à Sparte, in: Studi in onore di Arnaldo Biscardi, Bd. 2, Hrsg. F. Pastori (Milano 1982)

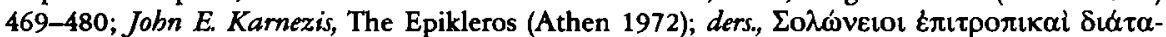
$\xi \varepsilon L_{5} . .$. (Athen 1976); W. K. Lacey, Die Familie im antiken Griechenland (Mainz 1983) [engl. London 1968]; Douglas M. MacDowell, Spartan Law (Edinburgh 1986); ders., The Oikos in Athenian Law, in: ClQu 39 (1989) 10-21; Alberto Maffi, Le mariage de la patrôoque adonnées dans le 
die Gäste umgelegt? Durch Zufall bin ich im nördlichen Burgenland ${ }^{1}$ auf eine Organisationsform gestoßen, die weitgehend ohne Geld auskommt. Nach dem Herkommen werden die Lasten des Festes von den Teilnehmern getragen, und zwar in natura: Rind, Schwein und Hühner werden nach Verwandtschaftsnähe beigesteuert, ebenso Schmalz, Mehl, Zucker, Eier, Nüsse, auch der Wein (nur die Schnāpse liefert der Wirt). Der Wirt stellt den Saal und verrechnet im wesentlichen nur Arbeitsleistung. Er ist hāufig Fachmann für das Schlachten und die Fleischbereitung. Gemeinsam mit der hochspezialisierten "Mehlspeisköchin" wandelt er in den Spuren der antiken Opferpriester.

Nach einem traditionellen Organisationsschema wird an solchen Festen eine gesellschaftliche Aufgabe bewāltigt, welche die wirtschaftlichen Kräfte des einzelnen Mitglieds bei weitem übersteigt. Das zeigt sich auch in Kleinigkeiten: In einem autarken Dorf ist es einer einzigen Familie praktisch unmöglich, zu einem bestimmten Zeitpunkt einige hundert frische Eier bereitzustellen. Also sammeln die geladenen Frauen zwei bis drei Wochen lang. Andererseits schafft es aber eine Hochzeitsgesellschaft erfahrungsgemäß nicht, die bei der Fleischbereitung anfallenden Suppen aufzuessen. Suppe wird schnell sauer. So besteht der Brauch, jenen nur entfernt Verwandten, die nicht zum Fest geladen sind, einen Topf Suppe ins Haus zu schicken. Als ich einmal

Code de Gortyne (col. VIII 20-30), in: RHD 65 (1987) 507-525; Joseph Modrzejewski, Zum hellenistischen Ehegüterrecht im griechischen und römischen Agypten, in: ZSStRom 87 (1970) 5084; ders., La structure juridique du mariage grec, in: Symposion 1979, Hrsg. P. D. Dimakis (Athen 1981 und Köln 1983) 39-71; Claude Mossé, La femme dans la Grèce antique (Paris 1983); dies, La contre Spoudias de Démosthène et les droits économiques des femmes d'Athènes, in: Symposion 1985, Hrsg. G. Thür (Köln-Wien 1989) 215-219; Ruth Oldenziel, The Historiography of Infanticide in Antiquity, in: Sexual Asymmetry, Hrsg. J. Blok, P. Mason (Amsterdam 1987) 87-107; Paul Roesch, Les femmes et la fortune en Béotie, in: La femme dans le monde méditerranéen, Bd. 1, Hrsg. A.-M. Vérilhac (Lyon 1985) 71-84; Barbara Röll, Untersuchungen zur privatrechtlichen Stellung der Frau in Böotien im 2.Jahrhundert vor Christus nach den Inschriften (Athen 1974); Hans-Albert Rupprecht, Zum Ehegattenerbrecht nach den Papyri, in: BASP 22 (1985) 291-295; ders., Ehevertrag und Erbrecht, in: Festschrift für Ramon Roca-Puig, Hrsg. S. Janeras (Barcelona 1987) 307-311; David M. Scbaps, Economic Rights of Women in Ancient Greece (Edinburgh 1979); Wolfgang Schuller, Frauen in der griechischen Geschichte (Konstanz 1985); Riet Van Bremen, Women and Wealth, in: Images of Women in Antiquity, Hrsg. $A$. Cameron, A. Kubrt (Detroit 1983) 223-242; Ronald F. Willetts, The Law Code of Gortyn (Berlin 1967); Hans Julius Wolff, Eherecht und Familienverfassung in Athen, in: Beiträge zur Rechtsgeschichte Altgriechenlands und des hellenistisch-römischen Agypten (Weimar 1961) 155-242 [urspr. in: Traditio 2 (1944) 43-95]; ders., Die Grundlagen des griechischen Eherechts, in: TR 20 (1952) 1-29, 157-163 [ = in: Zur Griechischen Rechtsgeschichte, Hrsg. E. Berneker (Darmstadt 1968) 620-654]; ders., Das attische Apotimema, in: Festschrift für Ernst Rabel, Bd. 2, Hrsg. W. Kunkel, H. J. Wolff (Tübingen 1954) 293-333; ders., Zur Geschichte der Parapherna, in: ZSStRom 72 (1955) 335-347; ders., $\pi$ poíz, in: RE 23/1 (1957) 133-170.

' Die altertümliche soziale Struktur der an der österreichisch-ungarischen Grenze gelegenen Gemeinde Tadten wurde vom Institut für Volkskunde der Universität Wien untersucht; Károly Gaál, Olaf Bockborn, Tadten. Eine dorfmonographische Forschung der Ethnographica Pannonica Austriaca (Wiss. Arb. aus dem Burgenland, Eisenstadt 1976), zur Hochzeit s. S. 258-264, zur „Hochzeitsköchin“ s. S. 190. Herr Prof. Gaál teilt mir freundlicherweise mit, daß das Hochzeitsmahl erst seit etwa 1970 im Gasthaus stattfindet, vorher wurde es im Eltemhaus der Braut veranstaltet. 
Zeuge solch einer Szene war, sagte die beschenkte Hausfrau beschāmt: „Eigentlich haben wir das ja nicht nötig, aber es ist bei uns so ein komischer Brauch.“

Damit bin ich beim Thema dieses Beitrags: Armut. Die soeben geschilderte Organisationsform ermöglicht es, soziale Aufgaben, in unserem Beispiel die Selbstdarstellung zweier Kleinfamilien als vollberechtigte Mitglieder der Dorfgemeinschaft, weitgehend unabhāngig von der konkreten Vermögenslage des einzelnen wahrzunehmen. Die kollektiven Mechanismen sind auf minimale wirtschaftliche Voraussetzungen zugeschnitten, eben Armut. Im folgenden will ich versuchen, solche Minimalvoraussetzungen im altgriechischen Familiengüterrecht aufzuspüren. Ist das Grundbedürfnis des einzelnen, genügend Nahrung zum Überleben zu finden, vielleicht der Schlüssel zu den rechtlichen Organisationsformen von Familienvermögen?

Lächerlich, kann man sofort einwenden, wenn man an das reiche Athen denkt, die großartige Seemacht des 5. und 4. Jahrhunderts, die Stadt mit den Prachtbauten auf der Akropolis, dem Kultbild der Athena Parthenos, üppig mit Gold (der Bundesgenossen) behängt; weit vom Meer her sah man die goldene Speerspitze der Athena Promachos leuchten. Diese Stadt war nicht arm. Dennoch war das Ehegüterrecht auf allerbescheidenste Verhältnisse zugeschnitten, die auch Athen lange genug erlebt hatte. Bescheidene Dorfsiedlungen waren die meisten griechischen Stadtstaaten, traurige, um das tägliche Brot kämpfende Nester, „Die letzte Welt“, wie sie Christoph Ransmayr in seinem Roman über Ovids Verbannungsort Tomi eingefangen hat. Es waren staatliche Organisationen, die in Kriegszeiten oft genug von einem Tag auf den anderen umgesiedelt wurden, die sich in Hungersnöten teilten und Oikisten - mit allen Segenswünschen geleitet und dem Abbild der staatlichen Ordnung in sich - wegschickten, wenn sie die zuviel geborenen Kinder nicht rechtzeitig ausgesetzt hatten ${ }^{2}$. Jedes Dorf war ein souveräner griechischer Staat, lehrte die Jugend (neben militärischer Disziplin) Homer, träumte von einem olympischen Sieger aus den eigenen Reihen, verehrte zu Hause, so gut es ging, die panhellenischen Götter und wünschte sich einen Tempel aus Stein anstatt des unansehnlichen Holzhauses - bezeichnenderweise waren im Stein- und Holztempel ohnehin die gleichen Konstruktionselemente und Maßverhältnisse verwirklicht.

Es wāre verwunderlich, wenn die Familienverfassung in den griechischen Poleis nicht ebenfalls auf gewisse einheitliche Konstruktionselemente zurückzuführen wäre. Hans Julius Wolff hat das in großen Aufsätzen (1961 [1944], 1952 und 1957) versucht. Moses I. Finley hat dem entschieden widersprochen ${ }^{3}$. Der theoretische Streit um die Einheit des griechischen Rechts oder die Vielfalt der griechischen Rechte, der gerade an dieser Frage entbrannte, ist hier nicht neu aufzurollen. Fest steht, daß die Quellen aus Athen und Gortyn, wo wir einigermaßen Bescheid wissen, aus Sparta, einigen Inseln und einigen Regionen des Mutterlandes sowie aus dem ptolemäischen Ägypten āußerlich ein recht buntes Bild bieten. Nicht ganz überzeugt bin ich freilich immer von den "Grundgedanken“, mit denen Wolff die verschiedenen Lösungen auf einen

${ }^{2} \mathrm{Zu}$ den Uberlebensstrategien der antiken Polis s. Garnsey, 43-86, bes. $64 \mathrm{f}$., und Oldenziel.

${ }^{3}$ Moses I. Finley, The Problem of the Unity of Greek Law, in: La storia del diritto nel quadro delle scienze storiche (Firenze 1966) $129 \mathrm{ff}$; dagegen Hans Julius Wolff, in: Symposion 1971, Hrsg. H. J. Wolff (Köln-Wien 1975) 20 ff. 
Nenner bringen oder auch differenzieren möchte. Doch wird sich sein aus der modernen Rechtsvergleichung entlehnter Ansatz weitgehend bestätigen. Meiner Ansicht nach hatte das klassische Athen mit dem Prinzip des einheitlichen, völlig in den Dienst der agnatischen Familiennachfolge gestellten Oikos und der für die Ehe wesentlichen Mitgift (Proix) die „rückständigste“ Familienverfassung. In dorischen Städten gab es hingegen keine vergleichbare Proix, doch es traten verschiedene Vermögensmassen in einer Familie auf, nämlich das staatlich zugeteilte Landlos, der Klaros, und das erworbene, frei vererbliche Vermögen. Das führt bei den Doriern, trotz politischer „Rückständigkeit“ gegenüber dem demokratischen Athen, zu einer gewissen rechtlichen Selbständigkeit der einzelnen Familienmitglieder, auch der weiblichen. In der hellenistischen Zeit sieht man neben der größeren individuellen Selbständigkeit eine Vielschichtigkeit von Vermögensmassen in der Familie, was aber Verfügungsbeschränkungen der einzelnen nach sich zieht. Die überholten Einrichtungen Oikos und Klaros sind verschwunden.

Im folgenden stütze ich mich hauptsächlich auf die Arbeiten Wolffs und seiner Schüler, Carl Cromme (1962, Delphi), Antonios Babakos (1966, Thessalien, und 1973, die Insel Kalymnos ${ }^{4}$ ) und Barbara Röll ${ }^{5}$ (1974, Böotien). Gewiß sind damit erst einige Ausschnitte des Themas vorbereitet; mehr als eine kurze Zusammenfassung kann ich hier nicht bieten. Wolff selbst hat das Thema Familienverfassung bis zu seinem Tod im Jahre 1983 nicht mehr verfolgt, da er seine Zeit in das Handbuch „Das Recht der Papyri“ investierte.

Für Athen kann ich mich in diesem Überblick auf das Allernotwendigste beschränken $^{6}$. Brennpunkt der Familienorganisation im klassischen Athen war der Oikos, was mit "Haus“ nur sehr unzulänglich, besser mit "Hof", einigermaßen treffend mit "familia" wiederzugeben ist. Der Oikos ist ein letztlich sakralrechtlich definierter Personenverband, der innerhalb des größeren Verbandes der Phratrie den Staat trägt ${ }^{7}$. Grundsatz des Erbrechts ist es, den Oikos von Generation zu Generation über legitime leibliche oder durch Rechtsakt bestimmte männliche Nachkommen fortzuführen. Ein wichtiges Argument in Erbschaftsreden ist häufig, der Oikos dürfe nicht „leer werden“

4 Zum Material von der Insel Tenos s. vorläufig Etienne.

$3 \mathrm{Zu}$ dem von Röll bearbeiteten Gebiet s. nun Roesch, bes. S. 76.

6 Harrison, 45-60 und 130-138, gibt eine insgesamt zuverlässige Darstellung.

7 Allzu scharf trennt MacDowell (1989) die drei Bedeutungen des Wortes: Haus, Vermögen und Familie. Wenig überzeugend sieht er dabei im „Vermögen“ die ältere Schicht; „Familie“ (als Personenverband) sei erst in der Rednerzeit hinzugekommen. Das vermutlich solonische Gesetz, wonach sich der Archon um ,leere Häuser“ kümmern müsse (Dem. 43,75), bezieht er (S. 19f.) auf Baulichkeiten. Verfehlt ist sein Argument, der Archon könne sich nicht gut um „nichtexistente Söhne“ kümmern: Da Athen die "posthume Adoption"kennt, kann das der Archon sehr wohl. Zu Unrecht schiebt er (S.10 Anm.2) auch den „Oikos der Dekeleier“" zur Seite (IG II ${ }^{2}$ 1237, 396/5 v. Chr.); in sakralem, sicher weit zurückweisenden Zusammenhang wird dort Oikos sogar für den Personenverband der Phratrie gebraucht, s. P. J. Rhodes, A. Commentary on the Aristotelian Athenaion Politeia (Oxford 1981) 69f. Berechtigte Kritik übt er (S. 20) an Wolff(1961 [1944]) 238[93], der die Polis als „Zusammenschluß von Oikoi“ betrachtet. Da aber normalerweise die durch Abstammung vermittelte Zugehörigkeit zu einem Oikos Voraussetzung für das Bürgerrecht ist, möchte ich an der staatstragenden Funktion des kleinsten Personenverbandes festhalten. 


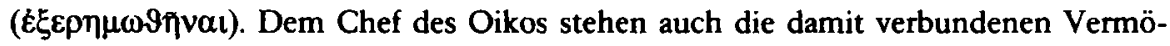
gensrechte zu. Ob durch Vermögensteilung unter mehreren Söhnen auch im sakralen Bereich mehrere Oikoi entstehen, kann ich nicht sagen. Hier scheinen Divergenzen zwischen Ideologie und wirtschaftlicher Realitāt zu klaffen, wie sie auch sonst in den Erbschaftsreden greifbar sind ${ }^{8}$. Daß Söhne zu Lebzeiten des Vaters am Vermögen beteiligt waren, scheint für Athen nicht belegt. Umgekehrt hat der Vater jedenfalls keinerlei Rechte am vom Sohn erworbenen Vermögen. Nur wenn der Sohn vor dem Vater stirbt, und zwar kinderlos, zeigt sich eine Besonderheit: Der Vater wird nicht Erbe seines Sohnes, sondern nimmt dessen Vermögen einfach an sich, so als ob eine latente Berechtigung auflebte?.

Welche Stellung haben Frauen in diesem System? Ublicherweise wechselt die legitime Tocbter in einen anderen Oikos über, um als Ehefrau den Fortbestand dieses Oikos zu sichern. Eigenartigerweise hat die Tochter, auch wenn sie noch Mitglied ihres ursprünglichen Oikos ist, kein Erbrecht nach dem Vater; daß die Ehefrau ihren Mann nicht beerbt, ist hingegen in einer patriarchalisch-agnatischen Familienverfassung nicht weiter verwunderlich. Frauen waren also notwendig, um das Erbrecht zu vermitteln, konnten in Athen aber niemals durch Erbrecht Vorstand eines Oikos werden. Demgemäß stand die Frau ihr Leben lang unter der personenrechtlichen Gewalt eines Kyrios: zunächst unter der Kyrieia des Vaters oder der Brüder, mit Verheiratung unter der des Ehemannes; dieser konnte sie testamentarisch weiterverheiraten, sonst wurden ihre Söhne Kyrioi oder die Kyrieia fiel an ihre Familie zurück. Auch wenn der Vorstand eines Oikos nur eine einzige Tochter hat, wird diese nicht Erbin. Als Erbtochter (Epikleros, „auf der Erbschaft sitzend“, wie Wolff in seinen Vorlesungen den Ausdruck anschaulich erklärte) fällt sie dem nächsten männlichen Agnaten, meistens also ihrem Onkel, als Ehefrau zu. Dieser zeugt mit ihr den männlichen Nachfolger seines Bruders: Erbe und Chef des Oikos wird dann erst der Enkel ${ }^{10}$.

In der bis jetzt skizzierten Familienverfassung hatte die Frau keine güterrechtlich relevante Stellung. Mit der Ehe verbunden waren aber zwei Arten von Vermögensverschiebungen: Seit altersher üblich, aber keineswegs notwendig, war die Pherné. Kleidung, Schmuck und Sachen zum persönlichen Gebrauch brachte die Frau in die Ehe mit. Bei Auflösung der Ehe gab es keine eigene Klage auf Herausgabe dieser Gegenstānde. Das besagt aber nicht, daß sie beim Mann geblieben wāren. Vielmehr wird die Frau ihre Sachen schlicht wieder mitgenommen haben. Daß sie in ihrem Eigentum standen, dürfte kaum zu bezweifeln sein. Solons Bemühungen, die Pherné auf ein bescheidenes Maß zurückzudrängen (Plut. Sol. 20,3 f.), sprechen für das hohe Alter dieser in den Quellen kaum erwähnten Einrichtung. Das Fehlen sonstiger rechtlicher Rege-

${ }^{8}$ Vgl. die fünf Oikoi der Erben des Buselos, Dem. 43,19; begreiflicherweise kommt hier auch MacDowell (1989), 16f. von seinem positivistischen Standpunkt her zu keinem Ergebnis.

9 Gegenargumente bringen Harrison, 138-141, MacDowell (1989), 13 Anm. 8. Streitigkeiten zwischen Vãtern und Söhnen über die Verwendung des „Familienvermögens“ waren nicht selten, s. Foxhall, 31 Anm. 47; Schlüsse auf gemeinschaftliche Verfügungsrechte sind hieraus jedoch nicht zu ziehen.

10 Zur Erbtochter in Athen s. Karnezis (1972 und 1976), Karabelias (1974) und die Beiträge von Eberbard Ruschenbusch, Alberto Maffi und Luisa Lepri Sorge, in: Symposion 1988, Hrsg. G. Nenci, G. Thür (Köln-Wien 1990). 
lungen zeigt entweder die geringe Bedeutung der Pherné im athenischen Ehegüterrecht oder daß sie keinen Anlaß zu Streit gab.

Fast untrügliches Zeichen für eine gültige Ehe war in klassischer Zeit jedoch eine Proix (Mitgift), die der Kyrios für das Mādchen bestellte. Die Proix war im 4.Jahrhundert üblicherweise eine Summe Geldes, die dem Ehemann ausgehändigt und durch ein Grundstück nach dessen Schätzung (Apotimema) gesichert wurde. Durch einen "Warnstein" kenntlich gemacht, war das Grundstück dem Zugriff anderer Gläubiger, aber auch des Staates entzogen. Unter diesen Umstānden ist die Frage, in wessen Eigentum die Proix während der Ehe stand, fast müßig. Selbstverständlich hatte der Mann die Verfügungsmacht über das Geld. Wolff (1957) 147 nimmt das auch für andere Gegenstände an, doch sprechen gewichtige Gründe dagegen ${ }^{11}$. Aus der Proix erhielt die in den Oikos eingetretene Ehefrau ihren Unterhalt. Bei Auflösung der Ehe kehrte die Frau wieder in ihren väterlichen Oikos zurück. Unabhängig davon, ob die Frau ein Verschulden am Scheitern der Ehe traf und ob sie Kinder im Hause des Mannes zurückließ, mußte dieser die Proix herausgeben. Waren aus der Ehe Kinder geboren, konnte die Frau nach dem Tode ihres Mannes aber auch in dessen Oikos verbleiben. In diesem Fall blieb auch die Proix dort. Starb die Frau kinderlos, ging die Proix zurück. Waren Kinder vorhanden, verblieb die Proix beim Mann und fiel nach dessen Tod als Sondervermögen den gemeinsamen Söhnen aus dieser Ehe zu.

Über den Sinn dieser Sondererbfolge an der Proix hat man sich schon seit langem Gedanken gemacht. Ludwig Mitteis ${ }^{12}$ hielt die Proix für einen der Tochter vorweg ausbezahlten Erbteil; Wolff widerlegt das mit dem Hinweis, daß es in Athen kein Erbrecht der Tochter gebe ${ }^{13}$. Er meint, daß durch die Proix den Söhnen der Frau ein Anteil am Vermögen der mütterlichen Familie gesichert worden sei, entsprechend dem auch durch die weibliche Linie vermittelten Erbrecht. Dem ist aber entgegenzuhalten, daß dieses Erbrecht in der weiblichen Linie erst dann zum Zuge kommt, wenn die männliche erschöpft ist (die Söhne der Brüder gehen also den Söhnen der Schwestern vor). Die Proix bleibt aber in jedem Fall in der weiblichen Linie.

11 Wolff folgen u. a. Schaps, 74ff., Schuller, $55 \mathrm{f}$., Mossé (1989), 215. Foxball, $34 \mathrm{ff}$. beruft sich für das Gegenteil vor allem auf Dem. 47, 57; überzeugend erklärt sie die Optik der Quellen, indem sie die Ebene der Familie von der der Polis trennt: Nach außen tritt nur der Kyrios der Frau in Erscheinung, innen kann eine mit entsprechender Proix ausgestattete Frau das Sagen haben. Freilich kann man aus der Drohung der Frau, mitsamt der Proix das Haus des Mannes zu verlassen, kein „Vetorecht“ (S.37) gegen unberechtigte Verfügungen des Mannes ableiten. Daß die Frau in Athen unter Kyrieia steht, betrifft nur ihre Geschäftsfähigkeit, nicht aber ihre Vermögensfähigkeit; für diese bringen Foxhall und Mossé eine Reihe von Belegen. Damit ist aber die Frage nicht gelöst, ob der Mann ohne Zustimmung der Frau Gegenstānde der Proix wirksam veräußern kann; Isai. 5,27 ist trotz Wolff(1957), 148 f. ein Sonderfall. Das Problem bleibt offen.

12 Reichsrecht und Volksrecht in den östlichen Provinzen des römischen Kaiserreichs (Leipzig 1891) $236 \mathrm{f}$., mit Hinweis auf Plat. Nom. $923 \mathrm{~d}$.

13 Wolff (1961 [1944]) 187 [62]; Foxball, 32 f. nähert sich wieder Mitteis, jedoch mit der unhaltbaren Behauptung, Töchter seien in Athen ebenso wie in Gortyn erbberechtigt gewesen (s. dazu weiter unten); auch daß der Wert der Proix üblicherweise dem Erbteil entsprochen habe, ist unrichtig, s. die Angaben bei Wolff(1957), 139 f. (wieder ist Isai. 5,27, worauf Foxball sich stützt, als Sonderfall auszuscheiden). 
Ich möchte demgegenüber eine einheitliche Erklārung vorschlagen, die an der Unterhaltsfunktion ansetzt. Kehrt die Frau nach Auflösung der Ehe in ihren vāterlichen Oikos zurück, steht ihrem Kyrios die Dike Proikos wegen Vorenthaltes der Mitgift zu. Daneben gibt es eine Dike Sitou (Getreide- oder Brotklage) auf den Betrag von jährlich $18 \%$ (oder monatlich 1,5\%) des Wertes der Mitgift. Wer die Mitgift in Händen hat, ist also zum Unterhalt der Frau verpflichtet. Eine mittelstāndische Proix von 20 Minen (2000 Drachmen) ${ }^{14}$ bringt also 30 Drachmen im Monat. Von einer Drachme am Tag kann im 4.Jahrhundert eine Person bequem leben. Aus dem Namen Sitou ergibt sich, daß der geschiedenen Frau ihr Unterhalt ursprünglich in natura zu leisten war, höchstwahrscheinlich aus dem Ertrag eines eigens als Proix bestellten Grundstücks. Aus dieser kleinlichen Regelung kann man schließen, daß es in manchen Familien problematisch gewesen sein mußte, einen zusätzlichen Esser, wie die zum Fortbestand der Familie notwendige Schwiegertochter, zu verkraften. Ebenso konnte die Rückkehr einer Tochter in das Haus des Vaters oder Bruders die übrige Familie in Verlegenheit bringen. So betrachtet ist die Proix in einer am unteren Ende des Nahrungsspielraums lebenden, seßhaften Ackerbaugesellschaft die nötige Konsequenz der agnatisch-patriarchalischen Familienverfassung: Die Proix sichert die systemerhaltende Beweglichkeit der Frauen von einem Oikos in den anderen - und notfalls auch wieder zurück. Bleibt die Frau jedoch plangemäß im Oikos ihres Mannes, so besteht auch über ihren Tod hinaus ein Bedarf an der zusätzlichen Nahrungsquelle. Denn aller Wahrscheinlichkeit nach hat die Familie einmal eine Tochter zu verheiraten. Das kostet ein Grundstück, während die Heirat eines Sohnes wieder eines einbringt. Würde jede Kleinfamilie nur einen Sohn und eine Tochter großziehen, könnte man sich das Hin und Her der Güter ersparen. Da trotz Familienplanung die Natur diesen Zustand des Gleichgewichts niemals erreicht, scheint das athenische System der Proix eine angemessene Lösung zur Sicherung des Existenzminimums der Kleinfamilien gewesen zu sein. Erbrechtliche Gründe für das Verbleiben der Proix in der Familie des Mannes muß man also nicht suchen ${ }^{15}$.

Daß die Bevölkerung Athens bittere Notzeiten erlebte, beweisen die Verhältnisse zur Zeit Solons. Die Schuldknechtschaft von Kindern, niemals aber der Ehefrau, zeigt, daß das Existenzminimum in einzelnen Familien oft genug unterschritten war $^{16}$. Zur Verarmung hatte vielleicht auch die Erbteilung geführt, die in Athen, soweit ersichtlich, niemals verboten war. Die krassen sozialen Gegensätze im 7. und 6. Jahrhundert erwecken deshalb $Z$ weifel daran, daß die Ideologie des Oikos sich damals auf die gesamte Bevölkerung erstreckte. Gewiß, das Geben und Nehmen von Mitgift mag auch in bescheidensten Verhältnissen noch funktioniert haben. Doch wer kümmerte sich darum, die Familie eines überschuldeten Kleinbauern fortzusetzen? Die kunstvollen

14 S. Schaps, 99, Appendix I; Wolff(1957), $139 \mathrm{f}$.

15 Daß ein Sohn in einem Antidosis-Verfahren erwägt, die Proix seiner im Hause lebenden Mutter von seinem eigenen Vermögen abzuziehen (Dem. 42,27), zeigt deutlich, daß man sich der getrennten Vermögensmassen stets voll bewußt war.

${ }_{16}$ Athen dürfte zur Zeit Solons noch nicht regelmäßig Getreide eingeführt haben, s. Garnsey, $110 \mathrm{f}$., der aber, wie Peter Herz, Rezension in: Gnomon 61 (1989) 138, einwendet, den ,subatlantischen Klimasturz" um 600 v. Chr. nicht berücksichtigt. Beides zusammen könnte die sozialen Probleme Athens gut erklären. 
Mittel wie testamentarische oder posthume Adoption und Erbtochterrecht scheinen der Aristokratie, und damit den Reichen, vorbehalten gewesen zu sein. Solon hat auch mit dem „Familienrecht“ seiner Gesetzgebung politischen Zündstoff beseitigt. Unter der Aufsicht des Archon eröffnete er jedem Bürger sāmtliche Möglichkeiten des Familienrechts, um sich den sakralen Fortbestand der eigenen Person durch legitime Nachkommen zu sichem. Wie andere Maßnahmen Solons trug auch diese zum sozialen Frieden bei, der die weitere, gedeihliche Entwicklung Athens kennzeichnete. Mit der Ideologie des Oikos trägt die Demokratie Athens aristokratische Züge. Auch der Kleinbürger hebt sich durch seine Abstammung von allen Nichtathenern ab, und er zeigt es ihnen, wenn er in der Volksversammlung oder zu Gericht sitzt. Ohne die von Armut (und Proix) mitgeprägte Organisation des Oikos wären freilich auch die unsterblichen Leistungen Athens, wäre seine Anmut nicht denkbar. Mit dem Ende der Demokratie - das hat schon Wolff (1952/1968) 163/654 vermutet - verschwindet diese ganz spezielle Ausprāgung einer griechischen Familienverfassung.

Das Recht Athens darf man nicht einfach verallgemeinem. Aus anderen, politisch unbedeutenden Städten sind uns auf Inschriften Splitter von Regelungen erhalten, die aber keine Schlüsse auf ein Gesamtkonzept zulassen. Am ehesten, jedoch noch weit mehr auf Hypothesen gestützt als meine Vermutungen zu Athen, lassen sich für die dorischen Staaten allgemeine Prinzipien des Familiengüterrechts erkennen. Hierzu muß man die lückenhaften, in sich oft widersprüchlichen Quellen aus Sparta mit den Aussagen der großen Gesetzesinschrift aus dem kretischen Gortyn aus der Mitte des 5. Jahrhunderts v. Chr. kombinieren.

Kennzeichen der dorischen Staaten ist es, daß eine dünne Erobererschicht über eine zahlenmäßig überlegene einheimische Bevölkerung herrscht. Sparta lebte theoretisch mit seinen Heloten in ständigem Kriegszustand. Sowohl die politische wie auch die Familienverfassung tragen dem dauernden militärischen Einsatz Rechnung. Vollberechtigter Spartiate und Mitglied der Mānnergemeinschaften, der Syssitien, war nur ein Besitzer eines der 9000 Landlose, eines Klaros. Dieses Los brachte 70 Medimnen für den Mann und 12 für die Frau ${ }^{17}$. Konsequenterweise war unter diesen Verhältnissen die Proix nicht nur überflüssig, sondern sogar verboten ${ }^{18}$. Ebenso konsequent dienten die Rechtseinrichtungen der Erbtochter und der Adoption dazu, für jeden Klaros stets mindestens einen waffenfähigen Inhaber zu stellen. Unter diesen Voraussetzungen hatte die Rechtseinrichtung der Erbtochter einen konkret faßbaren Zweck. Für die Zuteilung der Erbtöchter, für die Staatsstraßen und für Adoption seien die beiden Könige zuständig, schreibt Herodot ${ }^{19}$ ganz beiläufig. Selbstverständlich war der Klaros nach der Rhetra, der mündlich überlieferten, von dem sagenhaften Gesetzgeber Lykurg erlassenen „Verfassung“, unteilbar, unveräußerlich und unbelastbar. Das alles konnte aber den Niedergang des aristokratischen, in sich aber streng egalitāren Systems nicht aufhalten. Vermutlich bereits vor $404 \mathrm{v}$. Chr., also vor dem Ende des Peloponnesischen Krieges, hob Epitadeus das Veräußerungsverbot des Klaros auf; im

17 Plut. Lyk. 8,1,5-7; s. dazu MacDowell (1986), 89f., dem ich in diesem Abschnitt weitgehend folge.

18 Plut. Eth. 227 f.; MacDowell (1986), 81.

19 6,57,4-5; MacDowell (1986), $96 \mathrm{f}$. 
4. Jahrhundert seien, schreibt Plutarch ${ }^{20}$, von den 9000 Spartiaten nicht mehr als 700 übriggeblieben, von diesen hātten aber nur noch etwa 100 Land und Klaros besessen. In diesen Zusammenhang gehört die Bemerkung des Aristoteles ${ }^{21}:$ „Fast $z$ wei Fünftel des Landes gehört Frauen, weil die Epikleroi so viele geworden sind und weil man große Proikes gibt.“ Festzuhalten ist zunächst, daß hier die spartanischen Verhältnisse durch die Brille der athenischen Terminologie gesehen werden. Die Erbtochter heißt in Sparta nicht Epikleros, sondern Patroiouchos; auch der Ausdruck Proix ist im Dorischen unbekannt.

Um diese berüchtigte Aristoteles-Stelle und den Niedergang der spartanischen Familienverfassung zu deuten, scheint mir der Vergleich mit dem Gesetz von Gortyn hilfreich, der wichtigsten Rechtsquelle aus einer dorischen Stadt ${ }^{22}$. Die Bestimmungen enthalten auf den ersten Blick drei Paradoxa. Das erste: Eine Tochter kann einerseits erben, andererseits aber auch Erbtochter (Patroiokos) sein, d.h., sie vermittelt die Erbenstellung lediglich dem Enkel ihres Vaters. Das geht natürlich nicht an ein und derselben Vermögensmasse: Nicht erben kann die Tochter den Klaros, der die Mitgliedschaft in den Andreia (Speisegemeinschaften der Mānner) garantiert. Diese Stellung kann sie als Erbtochter nur vermitteln ${ }^{23}$. Eigenartigerweise ist im Gesetz die Nachfolge in den Klaros nicht direkt geregelt. Die erb- und familiengüterrechtlichen Bestimmungen sind nämlich alle auf das frei verfügbare Vermögen zu beziehen, das ein Bürger neben dem Klaros haben konnte. Dieses erben die Kinder folgendermaBen: Häuser und Vieh bekommen die Söhne, alles übrige wird dergestalt aufgeteilt, daß ein Sohn jeweils den doppelten Anteil einer Tochter bekommt (IV 31-38). Eine Erbtochter müßte demnach alleinige Erbin des freien Vermögens ihres Vaters werden. Verschmäht sie den nächsten Verwandten, der sie heiraten will, muß sie ihm das halbe Vermögen abtreten (VII 52 - VIII 8); daraus kann man schließen, daß es ihr, wenn sie plangemāß heiratet, zur Gānze verbleibt. Aber es steht dann in der Nutzung ihres Mannes (VI 10), und ihre Kinder haben ein latentes Recht daran (IV 22-27).

Ein zweites Paradoxon liegt darin, daß man in Gortyn - wie auch in Sparta - wegen des Klaros zwar auf eine Proix nicht angewiesen war, das Gesetz aber gleichwohl eheliche Zuwendungen genau regelt. Die Frau kann anläßlich der Eheschließung mit Vermögen „ausgesteuert“" werden, das aber ihren Erbteil aus dem freien Vermögen ihres Vaters nicht übersteigen darf (IV 48-51). Diese Zuwendung nimmt in der Tat die Erbschaft vorweg. Das Vermögen, das Matroion, bleibt stets Eigentum der Frau und fällt mit ihrem Tod (wieder im Verhältnis zwei zu eins) an die Söhne und Töchter aus dieser Ehe. Allerdings steht dem Ehemann über den Tod seiner Frau hinaus die lebenslängliche Kratesis (Nutzung) daran zu (VI 34). Nur wenn sich der Vater wiederverheiratet, geht die Kratesis schon vor dessen Tod auf die Kinder über (VI $44 \mathrm{f}$.).

\footnotetext{
20 Agis 5.

${ }^{21}$ Politik 1270a; beide Stellen sind ausführlich diskutiert von Christien, Karabelias (1982), Mac-

Dowell (1986), 99-110, Hodkinson.

22 Text und (mit Vorbehalt zu benützender) Kommentar bei Willetts.

${ }^{23}$ S. die spezielle Darstellung von Maffi mit reicher Bibliographie; s. a. Monika Lavrencic, Andreion, in: Tyche 3 (1988) 146-161.
} 
Wird die Ehe beendet, bekommt die Frau ihr Vermögen, die Hälfte der im letzten Jahr hieraus gezogenen Früchte und die Hälfte ihrer Webarbeiten heraus (II 45-52).

Eine derartige Aussteuer war jedoch, anders als in Athen, nicht geradezu zwangsläufiges Kennzeichen einer gültigen Ehe. Das beweist die Übergangsvorschrift V 1-9: Frauen, die vor einem bestimmten Datum ohne „Kremata“(Vermögen) geheiratet haben, haben auch jetzt keinen Anspruch darauf; die aber später geheiratet haben, können nun auf die Aussteuer klagen. Der frühere Rechtszustand erinnert an die Konzeption in Sparta: Der Klaros kann auch die Ehefrau mit ernähren. Offenbar reichten aber manche Klaroi in Gortyn nicht mehr aus, weshalb der Tochter ein vorweggenommener Erbanspruch am freien Vermögen ihres Vaters eingeräumt wurde. Möglicherweise eine dorische Spielart, Armut zu bewältigen.

Als drittes Paradoxon kann man anführen, daß es in Gortyn, ebenso wie in Sparta, keine Kyrieia über die Frau gab. Doch nützte ihr das nicht allzu viel: Das Vermögen, mit dem die Ehefrau vom Vater ausgesteuert wurde, stand, wie schon ausgeführt, in der Kratesis ihres Ehemannes; starb dieser vor ihr, trat ihr Sohn an dessen Stelle. Frei verfügen durfte sie nur über Kleinigkeiten (III 35). Immerhin konnte die Erbtochter sich weigern, den nächsten Verwandten zu heiraten. Die freie Wahl des Ehemannes mußte sie aber, wie bereits erwāhnt, vom Anspruchsberechtigten teuer erkaufen.

Nach diesem gedrängten Überblick über das relativ gut dokumentierte Regelwerk Gortyns kehren wir zurück in das dunkle Sparta. Möglicherweise hilft uns auch dort die Unterscheidung von Klaros und freiem Vermögen weiter. Wenn Aristoteles im 4.Jahrhundert sagt, Grund und Boden seien in der Hand von Erbtöchtern, so mag das wegen des Schwundes der männlichen Bevölkerung stimmen. Diesen alleinstehenden, ohne Chance auf einen ebenbürtigen Ehemann lebenden Frauen sind nicht nur - systemwidrig - die Klaroi ihrer Văter endgültig zugefallen, sondern ganz regulär auch deren freies Vermögen. Mißverständlich durch den attischen Sprachgebrauch ist vielleicht der Hinweis auf die Proix in Sparta. Aller Wahrscheinlichkeit nach handelt es sich dabei um Töchter jener reichsten Spartiaten, denen ihre Väter einen entsprechend hohen Erbteil vom freien Vermögen in die Ehe mitgegeben hatten.

Rückblickend kann man sagen, daß die Idee des dorischen Klaros und die darauf aufgebaute Familienverfassung vom Gang der Geschichte ebenso überholt wurden wie der Oikos Athens. Vor allem die Erbtochter verschwindet in der Folgezeit und macht im griechischen Kulturbereich einem allgemeinen Erbrecht der Tochter Platz ${ }^{24}$.

Nun komme ich allmählich in die Epoche, die als Vorstufe des byzantinischen Familienvermögens besonders interessant wäre, in die hellenistische Zeit, die im griechischen Osten des Reiches ohne großen Bruch in die römische übergeht. Doch muß ich mich hier auf eine knappe Zusammenfassung der eingangs erwähnten Vorarbeiten beschrānken. Klammert man die gut dokumentierte Praxis in Ägypten aus, bleibt nicht viel übrig. Bekanntlich wird die Mitgift, die den Charakter einer Abfindung des Tochtererbrechts erhält, in den Papyri bis in die römische Zeit hinein Pherné genannt, bis sich als Übersetzung von dos der Terminus Proix wieder einbürgert. Welche griechi-

24 Für die Papyri hat das bereits Hans Kreller, Erbrechtliche Untersuchung aufgrund der graecoaegyptischen Papyrusurkunden (Leipzig 1919) 143 ff., festgestellt. 
sche Rechtsordnung den Ausgangspunkt für die seit dem 2.Jahrhundert v. Chr. in den Papyri feststellbaren „Verfügungsgemeinschaften“ bildete, ist nicht geklärt. Es gibt zwar Untersuchungen über Pherné, Parapherna und Prosphora ${ }^{25}$, aber noch nicht über gemeinsame Verfügungen oder Mitberechtigungen am Familienvermögen, wovon etwa das Edict des Mettius Rufus aus dem Jahre $89 \mathrm{n}$. Chr. ausgeht ${ }^{26}$.

Aus dem Terminus Proix, der auf den Pfandsteinen (Horoi) von den Inseln Amorgos, Naxos und Syros und in den Mitgiftregistern von Mykonos und Tenos gebraucht wird, geht für das Ehegüterrecht genausowenig hervor wie aus dem aus Ephesos überlieferten Ausdruck Pherné27. Mehr Erfolg versprechen große Urkundenserien mit einheitlichen Geschäften, die zu Tausenden überlieferten Freilassungsurkunden. Üblicherweise wird der Sklave von einer Person oder mehreren an die Gottheit „verkauft", manchmal stimmen weitere Personen zu, oft werden noch Dienste vereinbart (Paramone) oder bei Sklavinnen die Stellung eines Ersatzkindes, schließlich wird noch das Erbrecht nach dem Freigelassenen geregelt. Aus der Beteiligung von Ehegatten, Kindern, Eltern an all diesen Geschäften und Berechtigungen kann man Schlüsse auf das Ehe- und Familiengüterrecht ziehen. So hat Cromme für Phokis aus den delphischen Inschriften der Jahre $200 \mathrm{v}$. Chr. bis $100 \mathrm{n}$. Chr. auf vertragliches kollektives Familieneigentum oder Gütergemeinschaft geschlossen. Babakos (1966) behandelte zunächst Thessalien, wo die Quellen bis in das 3.Jahrhundert n. Chr. fließen. Er stellt dort bei den Freilassungen ein eigenartiges Einspruchsrecht der Söhne, Töchter und Ehegatten fest, das er nicht als Miteigentum deutet, sondern als Paramone ex lege, den Rest einer personenrechtlichen Bindung des Sklaven an die gesamte Familie. Aus den ca. 60 Freilassungsinschriften der Insel Kalymnos aus dem 1.Jahrhundert n. Chr. leitet Babakos (1973) die volle Verfügungsfähigkeit der Frau ab, die keinen Kyrios brauchte. Wie in den Papyri konnte zwischen Eheleuten Verfügungsgemeinschaft vereinbart werden. Röll schließt aus den böotischen Texten auf die Erbberechtigung der Frau; Kinder hätten erbrechtliche Beispruchrechte, Ehegatten lebten eher in Güter- als in Verfügungsgemeinschaften, aber es gebe mütterliches und väterliches Sondervermögen.

Den Leser dieser Arbeiten beschleicht Unbehagen. Sind die Quellen nicht überfordert? Wird uns mehr Material aus anderen Landschaften weiterbringen? Jedenfalls sind die epigraphischen Zeugnisse noch durch die vom Rechtshistoriker bisher kaum bearbeitete hellenistisch-kaiserzeitliche Literatur anzureichern. Nützlich werden für diese Zeit auch die Ergebnisse der byzantinischen Rechtsgeschichte sein. Bis zum Beweis des Gegenteils würde ich davon ausgehen, daß sich die praktischen Probleme und die Versuche der Parteien, sie durch Vereinbarungen zu lösen, im griechischen Bereich durch die Jahrhunderte nicht grundlegend geändert haben. Vielleicht sollte man auch den Gesichtspunkt, den ich an die Spitze dieses Beitrags gestellt habe, mit berücksichtigen: Armut. Ehegüterrecht kann, wie auf dem Symposion immer wieder betont wurde, für die Witwe, die geschiedene Frau, die Kinder von existentieller Be-

\footnotetext{
${ }^{25}$ Zuletzt Häge, Modrzejewski (1970).

${ }^{26}$ Les lois des Romains, Bd. 2, Hrsg. P. F. Girard, F. Senn (Napoli ${ }^{7} 1977$ ), Nr. VII 9, Z. 34 f: Kratesis der Frau, Chresis der Kinder (Komm. Joseph Modrzejewski).

${ }^{27}$ Die Quellen sind bei $W$ olff (1957) am vollstāndigsten gesammelt.
} 
deutung sein. An die Stelle der globalen Lösungen der agnatischen Verwandtschaft, wie sie die klassische Polis bot, sind ab der hellenistischen Zeit vielschichtige Einzelvereinbarungen getreten. Die Inschriften belegen wenigstens das eine, daß in der Kleinfamilie selbstverständlich mehrere Vermögensmassen mit jeweils unterschiedlichen Bindungen existierten. Es wäre ein grober Fehler, vorschnell griffige dogmatische Lösungen anzubieten. 